\title{
AMELIORATIVE EFFECTS OF HESPERIDIN AND MELATONIN AGAINST ACETAMINOPHEN-INDUCED NEPHROTOXICITY IN ADULT ALBINO RATS
}

\author{
Hanan Tawfeek Emam, M.D. ${ }^{1}$ and Abdelmonem G. Madboly, M.D. ${ }^{2}$ \\ ${ }^{1}$ Department of Clinical Pharmacology, Faculty of Medicine, Benha University, Egypt \\ ${ }^{2}$ Department of Forensic Medicine and Clinical Toxicology, Faculty of Medicine, Benha \\ University, Egypt \\ Corresponding Author: Abdelmonem G. Madboly \\ Postal address: Faculty of Medicine, Benha University. Farid Nada Street, Benha City, Egypt. \\ Postal code: 13518 \\ Email: abdelmonem.algohari@fmed.bu.edu.eg \\ ORCID ID: https://orcid.org/0000-0002-4333-9018 \\ Mobile No: +201118734643 \\ Egypt J. Forensic Sci. Appli. Toxicol. Vol 21 (1) \\ Date of submission: 20 September 2020 \\ Revised at: 10 November 2020 \\ Accepted at: 19 November 2020
}

\begin{abstract}
Background: Acetaminophen (APAP) is the most widely used analgesic antipyretic drug, either by prescription or over the counter. APAP overdose can damage multiple organs, especially the liver and kidney, due to its toxic metabolites' formation and clearance. Aim: To investigate and compare the ameliorative effects of hesperidin (HSP) and melatonin (MEL) in rats with APAP-induced nephrotoxicity. Methodology: This comparative experimental study was carried out on 72 adult male Wistar albino rats were divided into nine groups (eight/each): groups (1-5): controls "negative, solvent, N-acetyl cysteine (NAC), MEL and HSP respectively; group (6): APAP-nephrotoxicity "rats were given a single dose $(900 \mathrm{mg} / \mathrm{kg})$ of APAP intraperitoneally (i.p.); group (7): APAP+NAC (150mg/Kg); group (8): APAP+MEL $(10 \mathrm{mg} / \mathrm{Kg})$ and group (9): APAP+HSP $(200 \mathrm{mg} / \mathrm{Kg})$. All treated drugs (NAC, MEL, and HSP) were given i.p. 30 minutes before APAP administration. Renal blood flow (RBF), blood urea, serum creatinine, tissue malondialdehyde (MDA), reduced glutathione (GSH), tumor necrotic factor alfa (TNF- $\alpha$ ), and interleukin-6 (IL-6) were estimated by colorimetric methods. Immunohistochemical expression of inducible nitric oxide synthase (iNOS) was detected, and histopathological examination of renal tissue specimens was performed. Results: HSP, MEL, and NAC partially improved APAP-induced nephrotoxicity, evidenced by biochemical, histopathological, and immunohistochemical findings. Conclusion: Both HSP and MEL alleviated APAP-induced nephrotoxicity; however, HSP was superior to MEL, and it was as equal as NAC (which was also superior to MEL) as regards the tested parameters. The ameliorative effects of HSP and MEL may be at least in part due to their antioxidant and antiinflammatory properties.
\end{abstract}

Keywords: Hesperidin, Melatonin, Acetaminophen, Nephrotoxicity, N-acetylcysteine.

\section{INTRODUCTION}

Acute renal injury is an alarming global public health issue affecting more than $10 \%$ of the whole population and constituting a major cause of morbidity and mortality worldwide (Hayek et al., 2020; Liu et al., 2020).
Drug-induced acute kidney injury (nephrotoxicity) can result from a predictable dose-dependent mechanism or an idiosyncratic reaction (Sales and Foresto, 2020). As the kidney is the major organ of drug biotransformation and excretion, it is at a high risk of damage due to the generation of reactive oxygen species 
(ROS) and toxic metabolites (Delavar and Soheilirad, 2020; Tjon and Teoh 2020). Acetaminophen (APAP) -the most common over-the-counter antipyretic, analgesic drug- is well known to cause either acute nephrotoxicity or renal failure in nearly $1-2 \%$ of APAP overdose cases, even in the absence of hepatic injury (Ilbey et al., 2009; Shen et al., 2020).

Toxic effects of APAP are mainly due to accumulation of ROS, such as nitric oxide (NO), peroxides, superoxide, etc., with resulting oxidative stress due to the formation of its toxic metabolite [N-acetylp-quinone imine (NAPQI)] (Chen et al., 2020; Mansourian et al., 2020).

Traditional treatment of APAP toxicity is done using $\mathrm{N}$-acetylcysteine (NAC), either by intravenous or oral regimens (Hendrickson, 2019). NAC is a potent antioxidant that encourages cellular glutathione (GSH) formation, attenuating NAPQI-mediated oxidative stress injury (Long et al., 2020).

However, NAC can result in several undesirable side effects, e.g., anaphylactoid reactions, seizures, gastrointestinal upset, cerebral edema, plus its low stability and bioavailability (Soliman et al., 2019; Firozian et al., 2020).

Hesperidin (HSP) is a safe and active type of bioflavonoids (secondary plant metabolites abundant in citrus species such as lemon, blood orange, orange, and lime). HSP has numerous useful pharmacological actions, e.g., antioxidant, anti-inflammatory, anticarcinogenic, antiviral, hypolipidemic, and hypoglycemic effects, without any side effects (Tabeshpour et al., 2020; Tao et al., 2020).

Melatonin (MEL) "N-acetyl-5-methoxy tryptamine" is a polypeptide derived from tryptophan, synthesized, and released by the pineal gland as the main hormonal controller of the circadian rhythm. Exogenous melatonin intake has immunostimulatory, anti-inflammatory properties, and antioxidant (direct free radical scavenger) activities, with a safe adverse-effect profile (Biancatelli et al., 2019; Pal et al., 2019).
Unfortunately, treatment of APAPinduced nephrotoxicity has not been wellestablished yet, as well as the molecular mechanism of APAP-induced nephrotoxicity is still unclear, so this work aimed to investigate and compare the ameliorative effects of hesperidin (HSP) and melatonin (MEL) against APAP-induced nephrotoxicity in adult male albino rats, as well as to illustrate their possible mechanisms of action. This may help to find an additional treatment strategy against APAP-induced nephrotoxicity.

\section{MATERIAL \& METHODS}

\section{Animals:}

Seventy-two adult male Wistar albino rats of a locally breaded strain weighing between 150-200 $\mathrm{g}$ at the beginning of the study were used. They were acclimatized for one week in groups (four/cage) in a fully ventilated room, under a $12 \mathrm{~h}$ light/dark cycle, at ordinary room temperature at the Pharmacology Department, Faculty of Medicine, with unlimited access to water and a balanced diet. All experimental protocols were conducted according to the local committee of the research ethics of Benha Faculty of Medicine.

\section{Drugs:}

Hesperidin (powder of purity $\geq 97.0 \%$, CAS No: 520-26-3), acetaminophen (powder, CAS No: 103-90-2), Melatonin (powder, CAS No: 73-31-4), NAcetylcysteine (powder, of purity $\geq 99.0 \%$, CAS No: 616-91-1) and polyethylene glycol (PEG)-400 (colorless, viscous, liquid of purity $>99 \%$, CAS No: 25322-68-3) were obtained from Sigma-Aldrich, Saint Louis, USA. Sterile Distilled water, diethyl ether, and formalin (neutral 10\% formalin) were obtained from Al-Gomhoria Pharmaceutical Chemical Co., Egypt. Chemicals and drugs were of a high analytical category and were freshly prepared before each experiment.

\section{Experimental design:}

In this comparative experimental study, and following acclimatization, animals were arranged into nine experimental groups (eight/each): 
Group I (negative control): animals in this group were kept in the same environment as the treated groups without any treatment to assess the tested parameters' normal values in this study.

Group II (solvent control): received drug vehicle [distilled water (four rats) or polyethylene glycol (PEG)-400] (four rats) via intraperitoneal (i.p.) injection in volumes comparable to that of tested drugs.

Group III (NAC control): treated with a single dose $[150 \mathrm{mg} / \mathrm{kg} / \mathrm{body}$ weight (b. wt.)] of $\mathrm{N}$-acetylcysteine (NAC) via i.p. injection, according to Sener et al. (2003).

Group IV (MEL control): treated with a single dose $(10 \mathrm{mg} / \mathrm{kg} / \mathrm{b}$. wt.) of melatonin (MEL) via i.p. injection, according to Sener et al. (2003).

Group V (HSP control): treated with a single dose $(200 \mathrm{mg} / \mathrm{kg} / \mathrm{b}$. wt.) of hesperidin (HSP) via i.p. injection (Ahmad et al., 2012).

Group VI (APAP): treated with a single dose $(900 \mathrm{mg} / \mathrm{kg} / \mathrm{b}$. wt.) of acetaminophen (APAP) via i.p. injection to induce nephrotoxicity, as described by Sener et al. (2003).

Group VII (APAP+NAC): received APAP and NAC in a dose-regimen, as illustrated in groups III and VI.

Group VIII (APAP+MEL): received APAP and MEL in a dose-regimen, as illustrated in groups IV and VI.

Group IX (APAP+HSP): received APAP and HSP in a dose-regimen, as illustrated in groups V and VI.

APAP, NAC, and MEL were dissolved in hot distilled water $\left(70^{\circ} \mathrm{C}\right.$, subsequently cooled to $37^{\circ} \mathrm{C}$ at treatment) (Sener et al., 2003); HSP was dissolved in PEG-400 (Anwer et al., 2014). All treated drugs (NAC, MEL, and HSP) were given 30 minutes before APAP administration (Sener et al., 2003).

After the end of the experiment (24 hours after APAP administration "the time needed to induce nephrotoxicity"), a renal incision was done under anesthesia (inhalation of ether) to expose the left kidney, and a bi-directional blood flowmeter
(Hadeco ES 1000 SPM, Japan) was fit in its artery to measure the renal blood flow, by setting the mode of pulsed doppler blood flowmeter, using ultrasonic gel on the probe top and turn the volume control to the maximum, then the probe pressed softly to the measurement area at an angle of $45-60^{\circ}$, and after hearing the optimal sounds for at least five seconds without moving the probe then press the freeze key to freeze the waveform (Haywood et al., 1981). After that, rats were sacrificed by decapitation under anesthesia. Blood samples were taken from abdominal aorta after dissection, and sera were obtained by centrifugation at 5000 round/minute for 5 minutes and kept at $-4 \mathrm{C}^{\circ}$. Kidneys were rapidly collected, washed with ice-cold saline, and longitudinally cut into two parts; one part was put in $10 \%$ formalin for immunohistochemical and histopathological examination, the other part was directly frozen at $-80 \mathrm{C}^{\circ}$, which latterly was minced and homogenized with tissue homogenizer, then it was centrifuged, and the resultant supernatant was used for assay of tissue oxidative stress markers.

\section{Studied Parameters:}

- Measurement of renal blood flow (RBF), using a doppler flowmeter (Hadeco ES 1000 SPM, Japan), according to Haywood et al. (1981).

- Biochemical measurements, using a spectrophotometer (model IL 143, Fischer Scientific, USA): (1984).

1- Blood urea, according to Kaplan

2- Serum creatinine, according to Murray (1984).

- Markers for oxidative stress:

1. Tissue reduced glutathione (GSH) was measured spectrophotometrically using commercial kits (Bio-diagnostic Co., Giza, Egypt), according to manufacturer's instructions (Asghar et al., 1975).

2. Tissue malondialdehyde (MDA) was measured spectrophotometrically using commercial kits (Bio-diagnostic Co., Giza, Egypt) according to the manufacturer's instructions (Yagi, 1984). 
3. Serum tumor necrotic factor alfa (TNF- $\alpha$ ) was measured by ELISA using commercial kits (USA \& Canada R\&D System, Inc) according to the manufacturer's instructions (Corti et al., 1992).

4. Serum interleukin-6 (IL-6) was measured by ELISA using commercial kits obtained from e-Bioscience (Vienna, Austria) according to the manufacturer's instructions (Klinik et al., 1998).

- Immunohistochemical expression of inducible nitric oxide synthase (iNOS):

Renal tissue sections ( $3 \mathrm{~mm}$ thickness) were moisturized in xylene and subsequently, in graded ethanol. After that, they were soaked in $5 \%$ bovine serum albumin (BAS) in Tris buffer saline (TBS) for 2 hours, then they were immune-stained with a primary antibody polyclonal immunoglobulin-G (IgG) of rat iNOS $(1 \mathrm{mg} / \mathrm{ml})$ with $5 \%$ BAS in TBS and incubated at $4^{\circ} \mathrm{C}$. Subsequently, they were incubated at room temperature with a secondary antibody, diluted 1:2000 with 5\% BAS in TBS for 2 hours. Lastly, sections were washed with TBS and incubated in a solution of $0.02 \%$ diaminobenzidine containing $0.01 \%$ hydrogen peroxide for 5 $10 \mathrm{~min}$. Staining was done by hematoxylin, and slides were examined using a light microscope [Olympus BX51, Hamburg, Germany] utilizing 10x and 40x magnifications (Ahmad et al., 2012).

Sections were graded as regard both the diffuseness and the intensity of 3,3'diaminobenzidine (DAB) staining as follows: zero (no staining), 1 [minimal (25\% staining)], 2 [mild (25\%-50\% staining)], 3 [moderate $(50 \%-75 \%$ staining) and 4 [marked (>75\% staining)]. Slides were tested by two separate examiners with no previous knowledge of the study protocol.

- Histopathological examination:

The kidney specimens were fixed in formalin $10 \%$, and histopathological examination was done using Hematoxylin and Eosin (H\&E) stain (Halliwell and Chirico, 1993). Statistical Analysis:
Obtained data were written as mean and standard deviation $( \pm \mathrm{SD})$; the one-way analysis of variance was used for data evaluation. The differences between groups were compared using Student's t-test, with $\mathrm{p}$ $<0.05$ was chosen as a statistical significance level (Khothari, 2004).

\section{RESULTS}

In the current work, negative, solvent, NAC, MEL, HSP control groups showed a non-significant difference as regard all studied parameters (nearly normal values as the negative control group), as well as sections (histopathological and immunohistochemical) from the kidney tissue, showed nearly normal structure as the negative control group, without any significant changes. So, they were represented in tables and figures as one group (controls).

Administration of a single i.p. dose of APAP $(900 \quad \mathrm{mg} / \mathrm{kg})$ caused marked impairment of renal function, as illustrated by significant $(\mathrm{p}<0.05)$ reduction in renal blood flow by $42.6 \%$, significant $(p<0.05)$ increase in blood urea and serum creatinine, renal MDA, serum TNF- $\alpha$ and IL- 6 by $162.8 \%, 229 \%, 426 \%, 119 \%, 345 \%$ respectively, with a significant $(\mathrm{p}<0.05)$ reduction in renal GSH by $62.2 \%$ compared with the control group (tables 1-3). This was associated with marked immunohistochemical expression of iNOS and histopathological changes in the form of loss of renal architecture (severe degeneration with dense lymphocytic infiltrate and marked necrosis), as illustrated in fig. 1-3. The administration of NAC revealed a significant $(\mathrm{p}<0.05)$ elevation in renal blood flow by $53.1 \%$, a significant $(p<0.05)$ decrease in blood urea and serum creatinine by $45.3 \%$, and $51.4 \%$ as compared with APAP-nephrotoxic rats. Renal MDA, serum TNF- $\alpha$ and serum IL-6 were significantly $(\mathrm{p}<0.05)$ reduced by $69.3 \%$, $31.5 \%$, and $49.6 \%$, respectively, with a significant $(\mathrm{p}<0.05)$ elevation of renal GSH by $138.7 \%$ as compared with APAPnephrotoxic rats (tables 1-3). This was 
associated with a moderate

the form of mild tubular vacuolation, and immunohistochemical expression of iNOS, inflammation (fig. 1-3).

moderate histopathological improvements in

Table (1): renal blood flow (RBF), blood urea, and serum creatinine levels in the different studied groups used to investigate and compare the ameliorative effects of hesperidin (HSP), and melatonin (MEL) in rats with APAP-induced nephrotoxicity (Mean $\pm \mathrm{SD}$ ):

\begin{tabular}{|c|c|c|c|}
\hline Proups & $\begin{array}{c}\text { RBF } \\
(\mathbf{c m} / \mathbf{s})\end{array}$ & $\begin{array}{c}\text { Blood urea } \\
(\mathbf{m g} / \mathbf{d l})\end{array}$ & $\begin{array}{c}\text { Serum creatinine } \\
(\mathbf{m g} / \mathbf{d l})\end{array}$ \\
\hline Controls & $9.4 \pm 0.73$ & $37.3 \pm 2.9$ & $0.42 \pm 0.02$ \\
\hline APAP group & $5.4 \pm 0.31^{\mathrm{a}}$ & $97.6 \pm 8.9^{\mathrm{a}}$ & $1.38 \pm 0.09^{\mathrm{a}}$ \\
\hline (APAP+ NAC) group $^{\text {aPAP+ MEL) group }}$ & $8.3 \pm 0.54^{\mathrm{a}, \mathrm{b}}$ & $53.4 \pm 4.6^{\mathrm{a}, \mathrm{b}}$ & $0.67 \pm 0.04^{\mathrm{a}, \mathrm{b}}$ \\
\hline (APAP+HSP) group $^{\text {APPP }}$ & $7.1 \pm 0.42^{\mathrm{a}, \mathrm{b}, \mathrm{c}}$ & $66.5 \pm 5.4^{\mathrm{a}, \mathrm{b}, \mathrm{c}}$ & $0.86 \pm 0.06^{\mathrm{a}, \mathrm{b}}$ \\
\hline
\end{tabular}

a: significant difference versus controls $(\mathrm{p}<0.05)$; b: significant difference versus APAP group $(\mathrm{p}<0.05)$; c: significant difference versus APAP+NAC treated group $(\mathrm{p}<0.05)$; $\mathbf{d}$ : significant difference versus APAP+ MEL treated group $(<0.05) ; \pm$ SD: standard deviation; APAP: acetaminophen; NAC: Nacetylcysteine.

Table (2): renal tissue reduced glutathione (GSH) and malondialdehyde (MDA) levels in the different studied groups used to investigate and compare the ameliorative effects of hesperidin (HSP), and melatonin (MEL) in rats with APAP-induced nephrotoxicity (Mean $\pm \mathrm{SD})$ :

\begin{tabular}{|c|c|c|}
\hline Groups & Renal GSH (nmol/g) & Renal MDA (nmol/g) \\
\hline Controls & $16.4 \pm 1.5$ & $0.73 \pm 0.06$ \\
\hline APAP group & $6.2 \pm 0.42^{\mathrm{a}}$ & $3.84 \pm 0.22^{\mathrm{a}}$ \\
\hline (APAP+ NAC) group & $14.8 \pm 0.91^{\mathrm{a}, \mathrm{b}}$ & $1.18 \pm 0.11^{\mathrm{a}, \mathrm{b}}$ \\
\hline (APAP+ MEL) group & $11,7 \pm 0.78^{\mathrm{a}, \mathrm{b}, \mathrm{c}}$ & $2.12 \pm 0.16^{\mathrm{a}, \mathrm{b}}$ \\
\hline (APAP+HSP) group & $15.2 \pm 1.12^{\mathrm{b}, \mathrm{c}, \mathrm{d}}$ & $1.15 \pm 0.09^{\mathrm{a}, \mathrm{b}, \mathrm{c}, \mathrm{d}}$ \\
\hline
\end{tabular}

a: significant difference versus controls $(\mathrm{p}<0.05)$; b: significant difference versus APAP group $(\mathrm{p}<0.05)$; $\mathbf{c}$ : significant difference versus APAP+ NAC treated group $(\mathrm{p}<0.05)$; $\mathbf{d}$ : significant difference versus APAP+ MEL treated group $(<0.05) ; \pm$ SD: standard deviation; APAP: acetaminophen; NAC: Nacetylcysteine. 
Table (3): serum tumor necrotic factor alfa (TNF- $\alpha$ ) and serum interleukin-6 (IL-6) levels in the different studied groups used to investigate and compare the ameliorative effects of hesperidin (HSP), and melatonin (MEL) in rats with APAP-induced nephrotoxicity (Mean \pm $\mathrm{SD})$ :

\begin{tabular}{|c|c|c|}
\hline Proups & TNF- $\alpha(\mathbf{p g} / \mathbf{m l})$ & IL-6 (pg/ml) \\
\hline Controls & $67.8 \pm 4.8$ & $200.2 \pm 13.2$ \\
\hline APAP group & $148.4 \pm 12.4^{\mathrm{a}}$ & $890.8 \pm 78.6^{\mathrm{a}}$ \\
\hline (APAP+ NAC) group & $101.6 \pm 8.2^{\mathrm{a}, \mathrm{b}}$ & $448.7 \pm 32.4^{\mathrm{a}, \mathrm{b}}$ \\
\hline (APAP+ MEL) group & $120.5 \pm 10.3^{\mathrm{a}, \mathrm{b}, \mathrm{c}}$ & $656.8 \pm 51.3^{\mathrm{a}, \mathrm{b}}$ \\
\hline (APAP+HSP) group & $103.2 \pm 8.4^{\mathrm{c}, \mathrm{d}}$ & $452.4 \pm 31.8^{\mathrm{a}, \mathrm{b}, \mathrm{c}, \mathrm{d}}$ \\
\hline
\end{tabular}

a: significant difference versus controls $(\mathrm{p}<0.05)$; b: significant difference versus APAP group $(\mathrm{p}<0.05)$; c: significant difference versus APAP+NAC treated group $(\mathrm{p}<0.05)$; $\mathbf{d}$ : significant difference versus APAP+ MEL treated group $(<0.05) ; \pm$ SD: standard deviation; APAP: acetaminophen, NAC: Nacetylcysteine.

The administration of melatonin (10 $\mathrm{mg} / \mathrm{kg})$ showed a significant $(\mathrm{p}<0.05)$ elevation in renal blood flow by $31.2 \%$, a significant $(\mathrm{p}<0.05)$ decreased in values of blood urea and serum creatinine by $32 \%$ and $37.7 \%$, respectively as compared with APAP-nephrotoxic rats. Renal MDA, serum TNF- $\alpha$ and serum IL-6 were significantly $(\mathrm{p}<0.05)$ reduced by $45 \%, 18.8 \%$, and $26.3 \%$, respectively, with a significant $(\mathrm{p}<0.05)$ elevation of renal GSH by $88.7 \%$ as compared with APAP-nephrotoxic rats (tables 1-3). This was also associated with a moderate immunohistochemical expression of iNOS and moderate histopathological improvements in the form of mild tubular vacuolation and inflammation (fig. 1-3).
On the other hand, administration of hesperidin $(200 \mathrm{mg} / \mathrm{kg})$ revealed a significant $(\mathrm{p}<0.05)$ elevation in renal blood flow by $63.2 \%$, a significant $(p<0.05)$ reduction in blood urea and serum creatinine by $49.1 \%$ and $53.6 \%$ respectively as compared with APAP-nephrotoxic rats. Renal MDA, serum TNF- $\alpha$ and serum IL-6 were significantly $(\mathrm{p}<0.05)$ reduced by $70 \%$, 30.5 , and $49.2 \%$, respectively, with a significant $(\mathrm{p}<0.05)$ elevation of renal GSH by $145.2 \%$ as compared with APAPnephrotoxic rats (tables 1- 3). This was associated with a mild immunohistochemical expression of iNOS and marked histopathological improvements correlated with biochemical and immunohistochemical results (fig. 1-3) . 

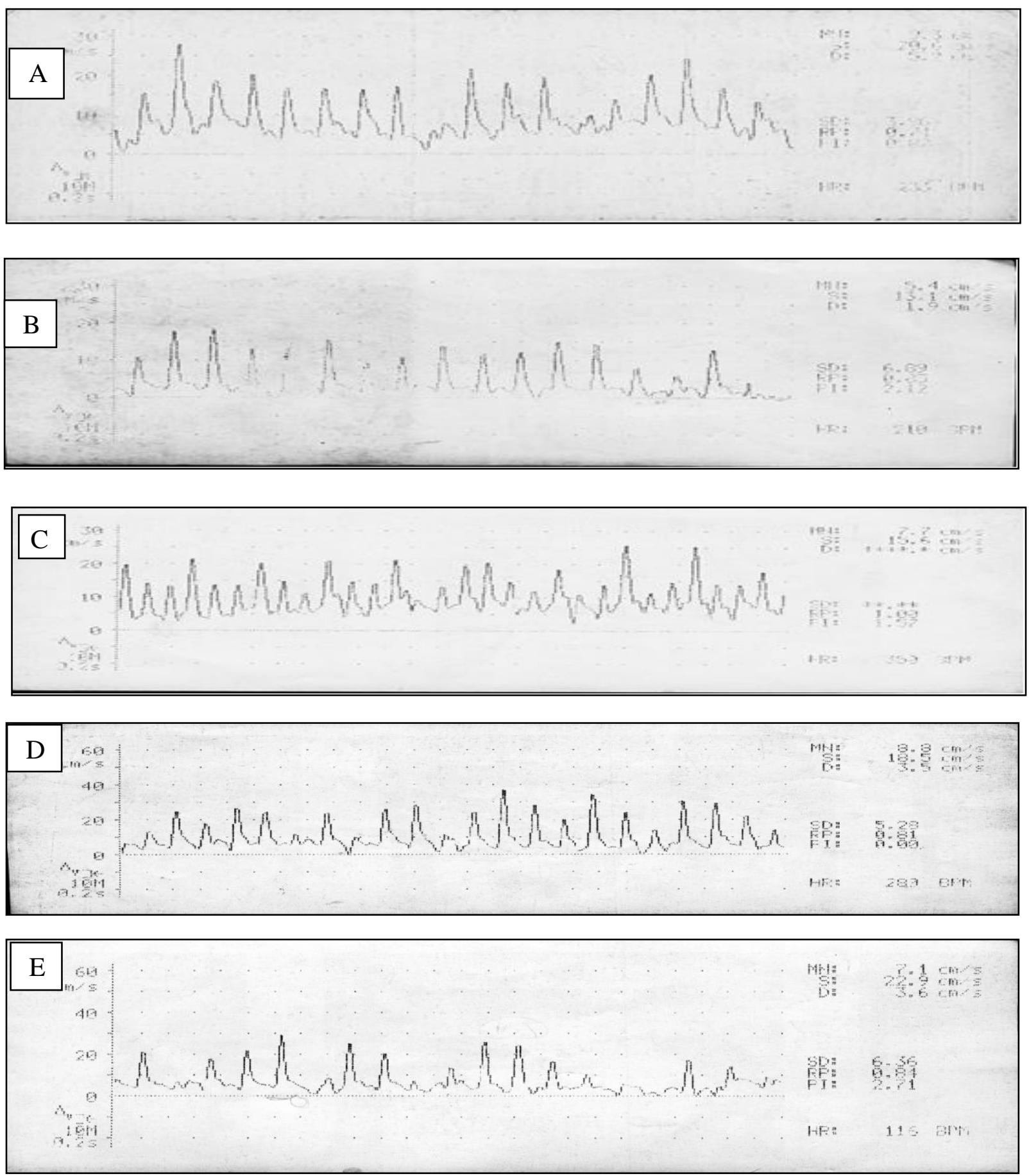

Figure (1): A trace (bi-directional blood flowmeter) showing renal blood flaw (RBF) in the different studied groups: (A): control rats (controls); (B): APAP-nephrotoxic rats (group 6); (C): APAP+NAC treated rats (group 7); (D): APAP+MEL treated rats (group 8); (E): APAP+HSP treated rats (group 9); [APAP: acetaminophen; NAC: N-acetylcysteine; MEL: melatonin; HSP: hesperidin]. 

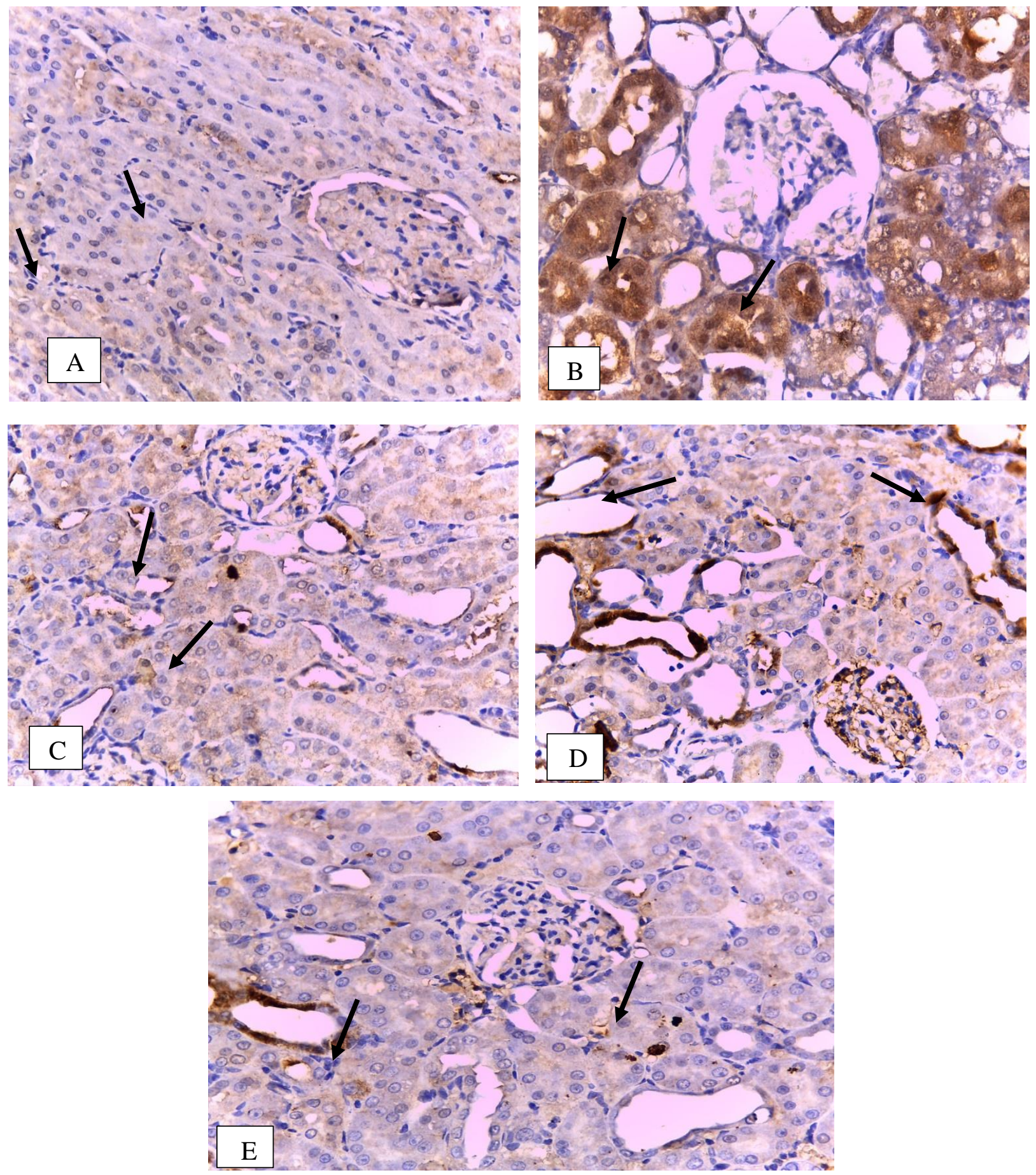

Figure (2): A photomicrograph illustrating the immunohistochemical staining of inducible nitric oxide synthase (iNOS) among the studied groups; (A): control rats (controls) showing minimal expression of iNOS; (B): APAP-nephrotoxic rats (group 6) showing marked expression of iNOS; (C): APAP+NAC treated rats (group 7) showing mild expression of iNOS; (D): APAP+MEL treated rats (group 8) showing moderate expression of iNOS; (E): APAP+HSP treated rats (group 9) showing mild expression of iNOS. [APAP: acetaminophen; NAC: N-acetylcysteine; MEL: melatonin; HSP: hesperidin] [Black arrows] (x400). 

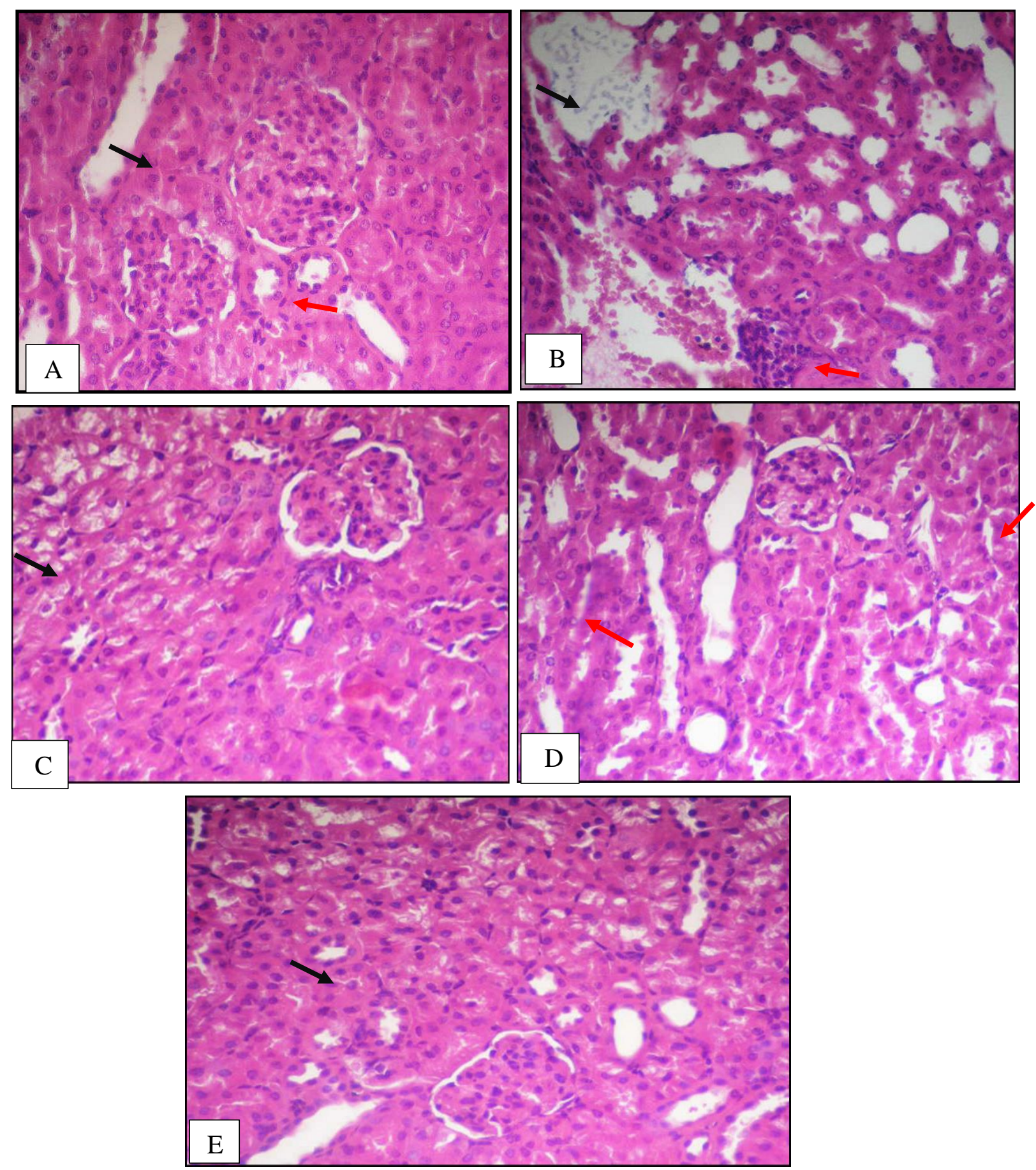

Figure (3): A photomicrograph of a transverse section of the kidney of (A): control rats (controls) showing central glomeruli surrounded by Bowman's space [black arrow], normal renal tubules lined by one layer of cuboidal epithelial cells [red arrow]; (B): APAPnephrotoxic rats (group 6) showing marked tubular necrosis [black arrow], interstitial congestion \& inflammation [red arrow]; (C): APAP+NAC treated rats (group 7) showing mild tubular vacuolation and inflammation [black arrow]; (D): APAP+ MEL treated rats (group 8) showing moderate tubular (vacuolation, congestion and inflammation) [red arrows]; (E): APAP+HSP treated rats (group 9) showing mild tubular vacuolation and inflammation with no congestion [black arrow]. [APAP: acetaminophen; NAC: Nacetylcysteine; MEL: melatonin; HSP: hesperidin] (H\&E X40). 


\section{DISCUSSION}

Overdose of acetaminophen (APAP) can produce hepatic and renal failure; the latter could occur in about 1-2\% of APAP overdose cases due to kidney damage because of oxidative stress (Ansari et al., 2020).

The results of this work revealed that i.p. administration of a single dose (900 $\mathrm{mg} / \mathrm{kg}$ ) of APAP resulted in nephrotoxicity, which was reflected by significant elevations in blood urea, serum creatinine, renal malondialdehyde (MDA), serum tumor necrotic factor alfa (TNF- $\alpha$ ), and serum interleukin-6 (IL-6) levels as well as significant reductions in renal blood flow (RBF) and renal reduced glutathione (GSH) with marked immunohistochemical expression of iNOS and the histopathological findings. These results were in line with those shown by Ilbey et al. (2009) and Molinas et al. (2010).

The mechanism of APAP-induced nephrotoxicity had gained a considerable argument. Conventionally, it is due to the production of NAPQI (a toxic metabolite) via cytochrome $\mathrm{P} 450$ enzyme in renal tissue. NAPQI depletes tissue GSH resulting in oxidative stress renal damage (Bessems and Vermeulen, 2001; Hilal et al., 2019).

Mazer and Perrone (2008) explained a different possible mechanism of APAP nephrotoxicity mediated by prostaglandinendoperoxide synthase (PGES), which can also transform APAP into NAPQI. This mechanism is more common in the renal medulla, while the cytochrome P450 is more pronounced in the renal cortex.

Ilbey et al. (2009) stated that the Ndeacetylase enzyme might also be responsible for APAP-induced nephrotoxicity, as it can convert either APAP itself or NAPQI to p-aminophenol, that is subsequently transformed to a free radical.

Despite the superfine variations, the endpoint of all these pathways is nearly typical (free radicals' formation), which resulted in oxidative stress tissue damage and necrosis. Such an assumption was confirmed by the significant increase in renal MDA (an end-product of free radical chain reaction) and a reduction in renal GSH (the main antioxidant). This was confirmed by the biochemical changes (elevated blood urea and serum creatinine) and the microscopic findings (hydropic degeneration and tubular necrosis).

Libby (2002) stated that inflammation and tubular necrosis might be explained by the pro-inflammatory effect of reactive free radicals, which induce the expression of leukocyte adhesive molecules, promoting leukocyte migration.

Free radical's formation promotes the expression of iNOS, cyclooxygenase-2 (COX-2), and the inflammatory cytokines such as TNF- $\alpha$, interleukin-1 (IL-1), and IL6 (Reuter et al., 2010).

Much more attention was made towards nitrosative stress or reactive nitrogen species (RNS), e.g., NO, and nitrogen dioxide in nephrotoxicity pathogenesis, a high level of NO production via iNOS is associated with an increased incidence of APAP-induced tissue inflammation and necrosis (Ito et al., 2003; Abdel-Zaher et al., 2008; Xie et al., 2016).

In this work and to prove this point, the results showed marked immunohistochemical expression of iNOS in immunohistochemical sections of the APAP-nephrotoxic group, mainly in renal tubules. This may be explained by the accumulation of the drug and its metabolites in the tubular cells, as previously confirmed by Morris and Levey (1994), who stated that renal excretion of acetaminophen in humans and animals involves glomerular filtration and passive reabsorption.

Treatment with either melatonin (MEL), hesperidin (HSP), or Nacetylcysteine (NAC) resulted in partial improvement of APAP-induced nephrotoxicity represented by significant elevation of RBF, significant reduction of blood urea and serum creatinine, and a significant decrease in renal MDA levels, serum TNF- $\alpha$ and serum IL-6 and increase in renal GSH with a significant reduction in 
iNOS expression, as compared with APAPnephrotoxic group.

This was in line with Sener et al. (2003), who reported that NAC, MEL, and vitamin E could ameliorate APAP-induced hepatotoxicity and nephrotoxicity, as they can scavenge free radicals.

Also, Ahmad et al. (2012) reported that HSP had hepato-protective and nephroprotective effects against APAPinduced toxicity and suggested that the antioxidant, anti-apoptotic and antiinflammatory properties of HSP as mechanisms of action.

These results were also in harmony with Subramanian et al. (2015), who studied HSP's ameliorative effect against gentamicin-induced nephrotoxicity and concluded that hesperidin possesses antiinflammatory properties and can significantly suppress TNF- $\alpha$ expression.

Renal tissue contents of MDA, GSH, and serum concentration of the inflammatory mediators (TNF- $\alpha$ and IL-6) were estimated as well as iNOS expression by immunohistochemical staining was recorded to explore the mechanism of the ameliorative effects of HSP and MEL against APAP-induced nephrotoxicity.

In the present study, both MEL and HSP showed a significant decrease in the renal tissue MDA, the serum concentration of TNF- $\alpha$ and IL- 6 levels, and the immunohistochemical iNOS expression with a significant increase of the renal GSH levels as compared to the APAP-nephrotoxic group.

The ameliorative effect of MEL against free radicals and oxidative stress is multifactorial. It can act as a direct free radical scavenger for hydroxyl free radical, singlet oxygen, and peroxy-radicals, and it can also inhibit the pro-oxidant pathway of nitric oxide synthase (NOS). Hydroxyl free radicals combine with $\mathrm{C}_{2}$ of the indole ring, forming 3-hydroxymelatonin, which is excreted in urine (Reiter et al., 2001\& 2016).

Moreover, Wang et al. (2004) and Rastogi et al. (2019) stated that MEL acts indirectly by stimulation of antioxidant enzymes; the most important of which is glutathione peroxidase, which induces the recycling of glutathione from inactive oxidized form to active reduced form, MEL has shown a great activity as a free radical scavenger in mitochondria which is the major intracellular site for electron leak that produces free radicals.

Hardeland and Pandi-Perumal (2005) stated that MEL could increase intracellular GSH concentrations by activating $\gamma$-glutamylcysteine synthase.

Also, Huang et al. (2007) and Koksal et al. (2012) showed that NAC and MEL had beneficial effects against hepatic injury in a rat model due to cardiopulmonary bypass and methanol toxicity, respectively; as serum levels of liver enzymes, TNF- $\alpha$, activities of iNOS, MDA, and myeloperoxidase in liver tissue were markedly reversed in both NAC and MEL groups. Furthermore, the glutathione content of liver and antioxidative enzyme activities markedly elevated after NAC or MEL treatment.

Ganeshpurkar and Saluja (2019) stated that HSP is a potent antioxidant, and it has an alleviative effect against druginduced nephrotoxicity, e.g., trichloroethylene and APAP nephrotoxicity rat models.

Ahmad et al. (2012) documented that HSP administration resulted in a significant reduction of iNOS expression in rats with APAP-induced nephrotoxicity.

Also, Pari et al. (2015) found that HSP significantly improved hepatic and renal functions, restored hepatic and renal antioxidants levels, and decreased the iron concentration in blood, suggesting antioxidant and heavy metal chelation mechanisms of HSP on iron-induced toxicity.

Moreover, Khan and Parvez (2015); Hajialyani et al. (2019) stated that HSP has a neuroprotective effect against the brain's toxic insults and neurodegenerative diseases, respectively, through amelioration of neuronal growth factors and antioxidant 
activities, as well as decreasing inflammation and apoptosis.

\section{CONCLUSION}

- A single i.p. dose $(900 \mathrm{mg} / \mathrm{kg})$ of acetaminophen (APAP) induced nephrotoxicity in rats, evidenced by biochemical, histopathological, and immunohistochemical findings.

- Hesperidin (HSP) "200 mg/kg"; melatonin (MEL) "10 $\mathrm{mg} / \mathrm{kg}$ " and $\mathrm{N}$ acetylcysteine (NAC) "100 mg/kg", given i.p. 30 minutes before APAP administration partially improved the picture of APAPinduced nephrotoxicity in rats.

- HSP was superior to MEL, and it was as equal as NAC (which was also superior to MEL) regarding the tested biochemical parameters.

- HSP and MEL's ameliorative effects effect may be at least in part due to their antioxidant and anti-inflammatory properties.

\section{RECOMMENDATIONS}

Further studies are needed to detect any untoward effects of hesperidin on other vital body functions in animal models and human beings with renal impairment. Moreover, the effect of a possible combination of hesperidin with other nephrotoxicityameliorative drugs commonly used should be considered.

\section{CONFLICT OF INTEREST (IF ANY)}

'The Authors declare that there is no conflict of interest.'

\section{ACKNOWLEDGMENT}

Many thanks to our colleges at the Faculty of Medicine, Benha University, for their valuable cooperation. http://www.fmed.bu.edu.eg

\section{REFERENCES}

Abdel-Zaher, A.O.; Abdel-Hady, R.H.; Mahmoud, M.M. and Farrag, M.M.Y. (2008): The potential protective role of alpha-lipoic acid against acetaminophen-induced hepatic and renal damage. Toxicol., 243 (3): 261-270. doi: 10.1016/j.tox.2007.10.010

Ahmad, S.T.; Arjumand, W.; Nafees, S.; Seth, A.; Ali, N.; Rashid, S.; et al. (2012): Hesperidin alleviates acetaminophen-induced toxicity in Wistar rats by abrogation of oxidative stress, apoptosis, and inflammation. Toxicol. Lett., 208: 149-161. doi: 10.1016/j.toxlet.2011.10.023

Ansari, S.; Azarmehr, N.; Barmoudeh, Z.; Moslemi, Z.; Ghahremani, H. Mirzaei, A.; et al. (2020): Evaluation of the protective potential of hydroalcoholic extract of Thymus daenensis on acetaminophen-induced nephrotoxicity in rats. Heliyon, 6: e03898. doi: 10.1016/j.heliyon.2020.e03898

Anwer, MK; Al-Shdefat, R.; Jamil, S.; Alam, P.; Abdel-Kader, M.S. and Shakeel, F. (2014): Solubility of bioactive compound hesperidin in six pure solvents at (298.15 to333.15) K. J. Chem. Eng. Data, 596: 2065-2069. doi: 10.1021/je500206w

Asghar, K.; Reddy, B.G. and Krishna, G. (1975): Histochemical localization of glutathione in tissues. J. Histochem. Cytochem., 23 (10): 774-779.

Bessems, J.G. and Vermeulen, N.P. (2001): Paracetamol (acetaminophen)induced toxicity: molecular and biochemical mechanisms, analogues, and protective approaches. Crit. Rev. Toxicol., 31 (1): 55-138. doi: 10.1080/20014091111677

Biancatelli, RMLC; Berrill, M.; Mohammed, Y.H. and Marik, P.E. (2019): Melatonin for the treatment of sepsis: the scientific rationale. J. Thorac. Dis., 12 (1): S54-S65. doi: 10.21037/jtd.2019.12.85 
Chen, H.; Wang, Y.; Jiao. F.; Yang. F.; Li, $X$. and Wang, L. (2020): Sinomenine attenuates acetaminophen-induced acute liver injury by decreasing oxidative stress and inflammatory response via regulating TGF- $\beta /$ Smad pathway in vitro and in vivo. Drug Design, Develop. Therap., 14: 23932403. doi:10.2147/DDDT.S248823

Corti, A.; Fassino, J. and Marcucci, F. (1992): Oligomeric tumor necrosis factor- $\alpha$ slowly converts into the reactive forms at bioactive levels. J. Biochem., 284: 905-910.

Delavar, M.A. and Soheilirad, Z. (2020): Drug and herbal medicine-induced nephrotoxicity in children; review of the mechanisms. J. Renal Inj. Prev., 9 (3): e21. doi: 10.34172/jrip.2020.21

Firozian, F.; Karami, S.; Ranjbar, A.; Azandaryani, M.T., and NiliAhmadabadi, A. (2020): Improvement of therapeutic potential Nacetylcysteine in acetaminophen hepatotoxicity by encapsulation in PEGylated nano-niosomes. Life Sci., 255: $117832 . \quad$ doi: 10.1016/j.lfs.2020.117832

Ganeshpurkar, A. and Saluja, A. (2019): The pharmacological potential of hesperidin. Ind. J. Biochem. Biophys., 56: 287-300.

Hajialyani, M.; Farzaei M.H.; Echeverria, J.; Nabavi, S.M.; Uriarte, E. and Sobarzo-Sanchez, E. (2019): Hesperidin as a neuroprotective agent: a review of animal and clinical evidence. Molecules. $24: \quad 648$. doi:10.3390/molecules24030648

Halliwell, B. and Chirico, S. (1993): Lipid peroxidation: its mechanism, measurement, and significance. Am. J. Clin. Nutr., 57: 715-724.

Hardeland, R. and Pandi-Perumal, S.R. (2005): Melatonin, a potent agent in antioxidative defense: actions as a natural food constituent, gastrointestinal factor, drug, and prodrug. Nutri. Metabol., 2 (22): $1-15$ doi:10.1186/1743-7075-2-22
Hayek, S.S.; Leaf, DE; Tahhan, A.S.; Raad, M.; Sharma, S.; Waikar, S.S.; et al. (2020): Soluble urokinase receptor and acute kidney injury. $\mathrm{N}$. Engl. J. Med., 382: 416-426. doi: 10.1056/NEJMoa1911481

Haywood, J.R.; Shaffer, R.A.; Fastenow, C.; Fink, G.D. and Brody, M.J. (1981): Regional blood flow measurement with pulsed doppler flowmeter in conscious rat. Am. J. Physiol., 241(2): H273-H278.

Hendrickson, R.G. (2019): What is the most appropriate dose of $\mathrm{N}$ acetylcysteine after massive acetaminophen overdose? Clin. Toxicol., 57 (8): 686-691. doi:10.1080/15563650.2019.1579914

Hilal, M.A.; Elsayed, R.M.; Mahmoud, S.F.; Abdallah, A.A. and Kasem, S.E. (2019): Ameliorative role of alpha lipoic acid on paracetamol induced renal toxicity and oxidative stress in rats. Egypt J. Forensic Sci. Appli. Toxicol.,
19
(1):
87-106.
doi:

10.21608/ejfsat.2019.7014.1044

Huang, H.; Yin, R,.; Zhu, J.; Feng, X,.; Wang, C,.; Sheng, Y.; et al. (2007): Protective effects of melatonin and $\mathrm{N}$ acetylcysteine on hepatic injury in a rat cardiopulmonary bypass model. J. Surg. Res., $142 \quad$ (1):153-161. doi: 10.1016/j.jss.2006.12.553

Ilbey, Y.O.; Ozbek, E.; Cekmen, M.; Somay, A.; Ozcan, L.; Otünctemur, A.; et al. (2009): Melatonin prevents acetaminopheninduced nephrotoxicity in rats. Int. Urol. Nephrol., 41(3): 695-702. doi: 10.1007/s11255-008-9503-z

Ito, Y.; Bethea, N.W.; Abril, E.R. and McCuskey, R.S. (2003): Early hepatic microvascular injury in response to acetaminophen toxicity. Microcirculation, 10: 391-400. doi: 10.1152/ajpgi.00217.2003

Kaplan, A. (1984): Urea. Kaplan. Clinical Chemistry. The C.V Mosby Co. St Louis Toronto Princeton. 1257-1260 and 437 and 418. 
Khan, M.H.A., and Parvez, S. (2015): Hesperidin ameliorates heavy metalinduced toxicity mediated by oxidative stress in brain of Wistar rats. J. Trace Elem. Med. Biol., 3: 53-60. doi: 10.1016/j.jtemb.2015.03.002

Khothari, C.R. (2004): Research Methodology: Methods and Techniques, New Age International, New Delhi.

Klinik, M.; Muller, A.M. and Rose-John, S. (1998): Interleukin-6 and soluble interleukin-6 receptor: direct stimulation of gp130 and hemopoiesis. Blood, 15 (29): 3495-3504.

Koksal, M.; Kurcer, Z.; Erdogan, D.; Iraz, M.; Tas, M.; Eren, MA.; et al. (2012): Effect of melatonin and $\mathrm{N}$ acetylcysteine on hepatic injury in rat induced by methanol intoxication: a comparative study. Eur. Rev. Med. Pharmacol. Sci., 16: 437-444.

Libby, P. (2002): Inflammation in atherosclerosis. Nature; 420 (6917): 868-874. doi: 10.1038/nature01323

Liu, D.; Cheng, F.; Pan, S. and Liu, Z. (2020): Stem cells: a potential treatment option for kidney diseases. Stem Cell Research Therap., 11 (249):1-20. doi: 10.1186/s13287-020-01751-2

Long, X.; Song, J.; Zhao, X.; Zhang, Y.; Wang, H.; Liu, X.; et al. (2020): Silkworm pupa oil attenuates acetaminophen-induced acute liver injury by inhibiting oxidative stressmediated NF-Kb signaling. Food Sci. Nutr., 8: 237-245 doi: 10.1002/fsn3.1296

Mansourian, M.; Mirzaei, A.; Azarmehr, N.; Vakilpour, H.; Kokhdan, E.P. and Doustimotlagh, A.H. (2020): Hepatoprotective and antioxidant activity of hydroalcoholic extract of Stachys pilifera. Benth on acetaminophen-induced liver toxicity in male rats. Heliyon, 6: e03029. doi: 10.1016/j.heliyon.2019.e03029

Mazer, M. and Perrone, J. (2008): Acetaminophen-induced nephrotoxicity: pathophysiology, clinical manifestations, and management. J. Med. Toxicol., 4 (1): 26.

Molinas, SM; Rosso, M.; Wayllace, N.Z.; Pagotto, M.A.; Pisani, G.B.; Monasterolo, LA.; et al. (2010): Heat shock protein 70 induction and its urinary excretion in a model of acetaminophen nephrotoxicity. Pediatr. Nephrol., 25 (7): 1245-1253. doi: 10.1007/s00467-010-1493-2

Morris, M.E., and Levy, G. (1994): Renal clearance and serum protein binding of acetaminophen and its major conjugates in humans. J. Pharm. Sci., 73 (8): 10381041. doi: 10.1002/jps.2600730806

Murray, R.L. (1997): Creatinine. Kaplan A. et al. (eds), Clinical Chemistry. The C.V Mosby Co. St Louis Toronto Princeton. 1261-1266 and 418.

Pal, P.K.; Bhattacharjee, B.; Chattopadhyay, A. and Bandyopadhyay, D. (2019): Melatonin as an armament against non-steroidal anti-inflammatory drug (NSAID) induced gastric injury: An overview. Melatonin Res., 2 (1): 115-137. doi: 10.32794/mr11250015

Pari, L.; Karthikeyan, A.; Karthika, P. and Rathinam, A. (2015): Protective effects of hesperidin on oxidative stress, dyslipidaemia and histological changes in iron-induced hepatic and renal toxicity in rats. Toxicol. Rep., 2: 46-55. doi: 10.1016/j.toxrep.2014.11.003

Rastogi, S.; Gupta, S.; Haldar, C. and Chandra, D. (2019): Nephroprotective effect of melatonin and Lascorbic acid (Vitamin-C) against ampicillin-induced toxicity in Funambulus pennanti. Egyp. J. Basic Appl. Sci., 7 (1): 8-19. doi:10.1080/2314808X.2019.1707626

Reiter, R.J.; Mayo, J.C.; Tan, D.; Sainz, R.M.; Alatorre-Jimenez, M. and Qin, L. (2016): Melatonin as an antioxidant: under promises but over delivers. J. Pineal Res., 61: 253-278. doi: 10.1111/jpi.12360

Reiter, R.J.; Tan, D.; Manchester, LC, and Qi, W. (2001): Biochemical 
reactivity of melatonin with reactive oxygen and nitrogen species. Cell Biochem. Biophys, 34: 237-256. doi: 10.1385/CBB:34:2:237

Reuter, S.; Gupta, S.C.; Chaturvedi, MM, and Aggarwal, B.B. (2010): Oxidative stress, inflammation, and cancer: How are they linked? Free Rad. Biol. Med., 49 (11): 1603-1616. doi: 10.1016/j.freeradbiomed.2010.09.006

Sales, GTM, and Foresto, R.D. (2020): Drug-induced nephrotoxicity. Rev. Assoc. Med. Bras., 66 (1): S:82-S90. doi: 0.1590/1806-9282.66.S1.82

Sener, G.; Sehirli, A.O. and AyanoğluDülger, G. (2003): Protective effects of melatonin, vitamin $\mathrm{E}$ and $\mathrm{N}$ acetylcysteine against acetaminophen toxicity in mice: a comparative study. J. Pineal Res., 35 (1): 61-68.

Shen, Y.; Jin, X.; Chen, W.; Gao, C.; Bian, Q.; Fan, J.; et al. (2020): Interleukin-22 ameliorated acetaminophen-induced kidney injury by inhibiting mitochondrial dysfunction and inflammatory responses. Appl. Microbiol. Biotechnol., 104: 58895898. doi:10.1007/s00253-020-10638-4

Soliman, A.A.I.; Fouda, A.; Metwally, E.S.; Madboly, A.G. and Hindawy, R.F. (2019): Ameliorative effect of cimetidine and silymarin on acute acetaminophen-induced hepatotoxicity in adult albino rats: an experimental comparative study. Egypt J. Foren. Sci. Appli. Toxicol., 19 (2): 101-120. doi: 10.21608/ejfsat.2019.7157.1047

Subramanian, P.; Anandan, R.; Jayapalan, J.J. and Hashim, O.H. (2015): Hesperidin protects gentamicin- induced nephrotoxicity via $\mathrm{Nrf} 2 / \mathrm{HO}-1$ signaling and inhibits inflammation mediated by $\mathrm{NF}-\kappa \mathrm{B}$ in rats. J. Funct. Foods, 13: 89-99. doi: 10.1016/j.jff.2014.12.035

Tabeshpour, J.; Hosseinzadeh, H.; Hashemzaei, M. and Karimi, M. (2020): A review of the hepatoprotective effects of hesperidin, a flavanon glycoside in citrus fruits, against natural and chemical toxicities. DARU J. Pharm. Sci., 28: 305-317. doi: 10.1007/s40199-020-00344-x

Tao, G.; Dagher, F.; Moballegh, A. and Ghose, R. (2020): Role of oxidative stress in the efficacy and toxicity of herbal supplements. Curr. Opinion Toxicol., $20 \quad$ (21): $36-40$. doi:10.1016/j.cotox.2020.04.004

Tjon, J. and Teoh, CW (2020): Medication-induced nephrotoxicity in children. Curr. Pediatr. Rep., 8:122133. doi:10.1007/s40124-020-00223-8

Wang, H.; Wei, W.; Shen, Y.X.; Dong, C.; Zhang, L.L.; Wang, N.P.; et al. (2004): Protective effect of melatonin against liver injury in mice induced by Bacillus Calmette-Guerin plus lipopolysaccharide. World J. Gastroenterol., 10 (18): 2690-2696.

Xie, W.; Wang, M.; Chen, C.; Zhang, X. and Melzig, MF. (2016): Hepatoprotective effect of isoquercitrin against acetaminophen-induced liver injury. Life Sci., 152: 180-9. doi: 10.1016/j.lfs.2016.04.002

Yagi, D.F.; Beutler, J.A., and Aust S.D. (1984): Microsomal lipid peroxidation. Methods Enzymol., 52: 302-311. 


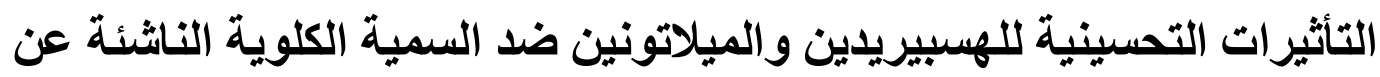

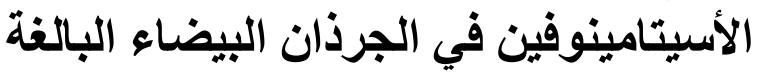

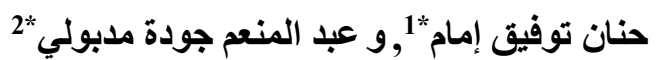

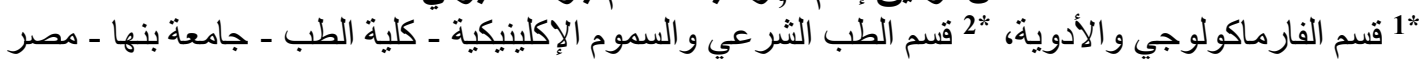

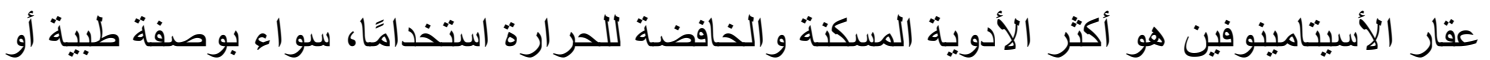

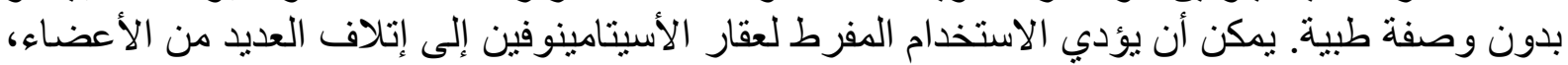

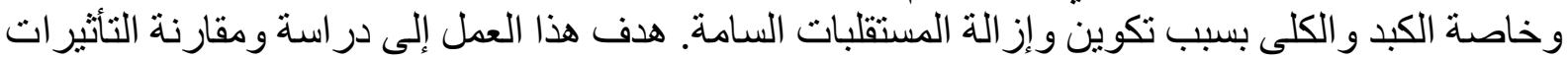

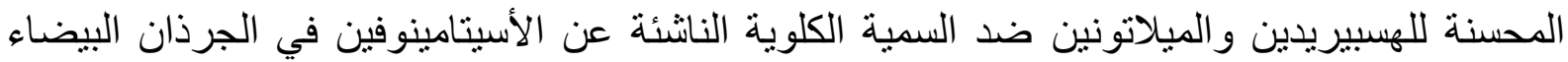

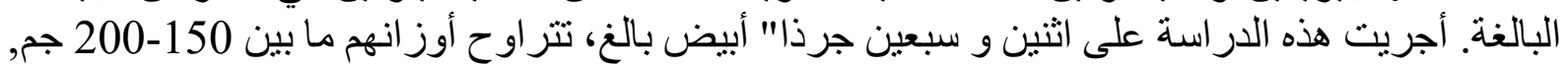

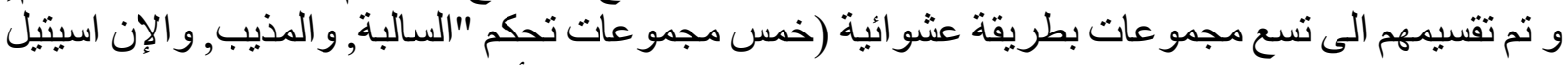

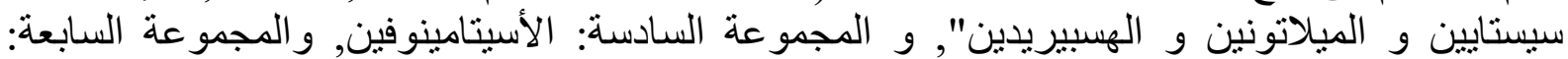

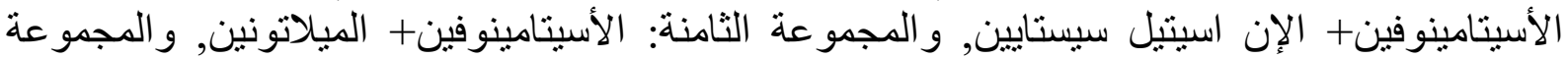

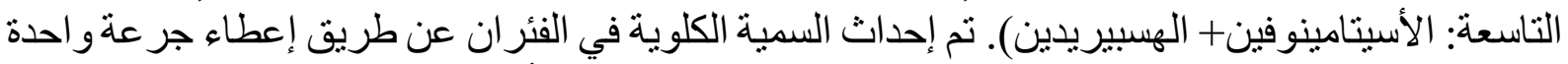

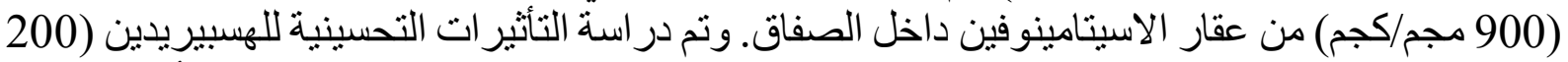

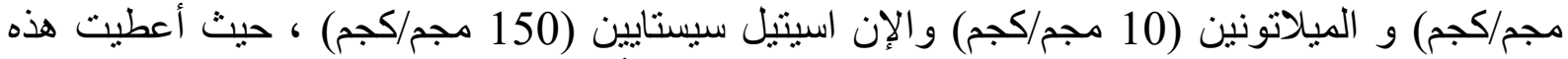

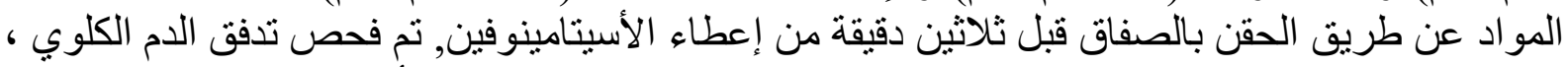

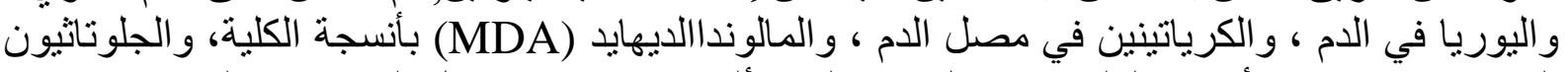

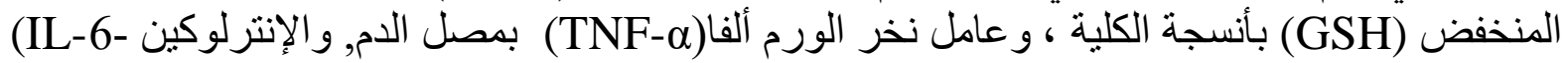

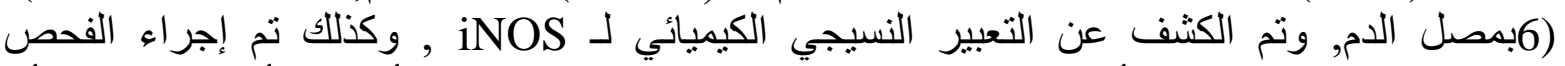

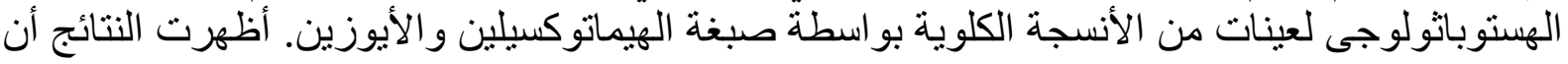

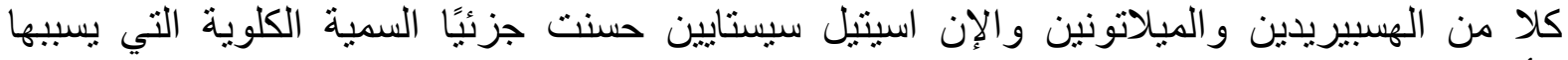

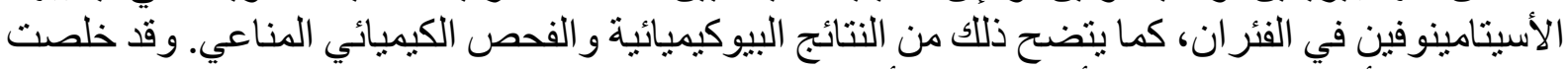

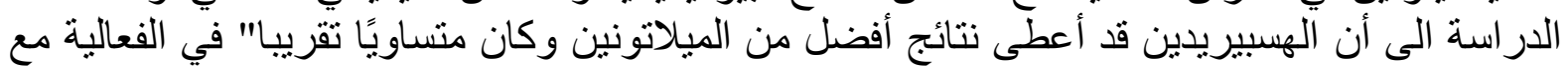

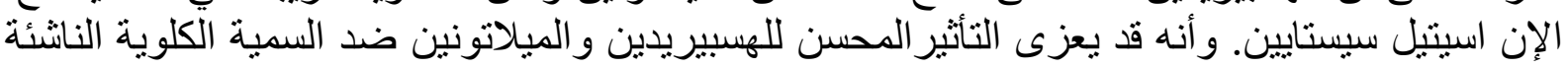

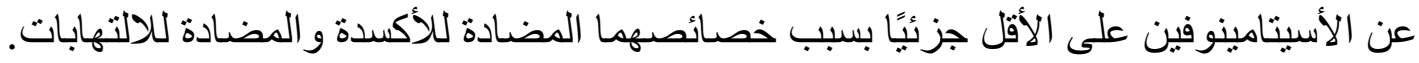

